\title{
Single electromagnetic sub-cascades in hadronic showers as the background in IACT observations
}

\section{Dorota Sobczynska}

Department of Astrophysics, University of Łódź, Pomorska 149/153, 90-236 Łódź,Poland E-mail: ds@kfd2.phys.uni.lodz.pl

The Air Cherenkov telescope may be triggered by light produced by charged particles in the single electromagnetic sub-cascade, which is a part of hadron induced shower. When image contains photons from only a single electromagnetic sub-cascade, it has similar shape to the gamma images. This effect results in the lowering of the gamma/hadron separation efficiency. The Monte Carlo simulations shown that the fraction of events containing Cherenkov light from one gamma sub-cascade in the total hadronic background depends on the trigger threshold and the observation mode

25th Texas Symposium on Relativistic Astrophysics - TEXAS 2010

December 06-10, 2010

Heidelberg, Germany 


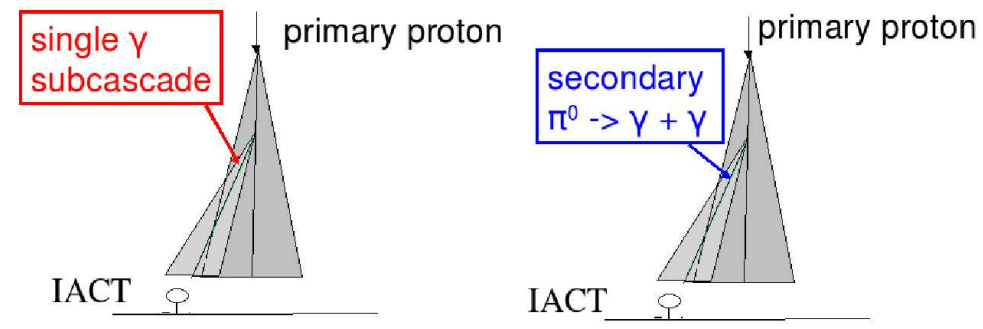

Figure 1: False $\gamma$ and one $\pi^{0}$ event

\section{Introduction}

It has been shown that one large Imaging Air Cherenkov telescope or system of two large IACT's, may trigger by Cherenkov photons produced by a single electromagnetic (em) sub-cascade in proton induced shower [1, 2, 的 (see Figure 1). There is no physical reason for differences in shapes of this kind of images and primary gamma images. The background from single em. subcascades and one $\pi^{0}$ events (two electromagnetic sub-cascades which are the products of one $\pi^{0}$ decay) seems to be one of main reasons of worsening the sensitivity of the IACT experiments. The results presented here show that even in case of three IACT working in coincidence mode one may expect not negligible fraction of single sub-cascades events in the detected hadronic background. All results presented here are based on Monte Carlo simulations.

\section{Monte Carlo Simulations}

The CORSIKA code version 6.023 [4, 可] has been used for the Monte Carlo simulations. The standard CORSIKA code was modified to keep additional information about each electromagnetic sub-cascade produced in the Extensive Air Shower (EAS). The simulations have been done for the MAGIC site [6, 7, 8, 9], that is $2200 \mathrm{~m}$ above sea level (around $800 \mathrm{~g} / \mathrm{cm}^{2}$ ). The night sky background (NSB) which was measured on La Palma [10], was included in the simulations as well as the Rayleigh and Mie scattering of light in the atmosphere (according to the Sokolsky formula [11]). The four MAGIC - like telescopes was chosen as an example of the system of large IACT. Telescopes were located in corners of a diamond with the length of sides $85 \mathrm{~m}$ and diagonals of $85 \mathrm{~m}$ and $147 \mathrm{~m}$. The diameter of each telescope's mirror dish is $17 \mathrm{~m}$. The total reflector area of one telescope covers more than $230 \mathrm{~m}^{2} .22$ million of showers initiated by primary protons with energies between $30 \mathrm{GeV}$ and $1 \mathrm{TeV}$ following a power law with a differential spectral index of -2.75 were simulated. The impact parameter was distributed randomly within a circle (radius of 1.2 $\mathrm{km}$ on the plane perpendicular to the shower axis) around the centre of the telescope system. The showers were simulated within a cone with an opening angle of $5.5^{\circ}$ at a zenith angle of $20^{\circ}$ and an azimuth of $0^{\circ}$ (showers directed to the north). For the primary $\gamma$ simulations (in total 1 million) the impact parameter was randomly distributed within a circle of $350 \mathrm{~m}$ radius with an energy range of $10 \mathrm{GeV}$ to $1 \mathrm{TeV}$. The differential spectral index was chosen to be -2.6 (which is the index of 
the Crab spectrum for energies above $300 \mathrm{GeV}$ or $500 \mathrm{GeV}$ [12, 13]. The results presented in this paper were obtained for a system of IACTs with a $3 \mathrm{NN}$ (next neighbouring pixels) trigger logic condition and trigger thresholds: 2, 3, 4 and 5 photoelectrons (p.e.).

\section{Results and Discussion}

I considered three possible operation modes: the only one (mode I), the only two (mode II) and the only three (mode III) telescopes fulfilled trigger conditions. Figure 1 shows the number of electromagnetic sub-cascades contributing to shower images versus the proton primary energy for trigger threshold of 3 p.e.. Distributions of number of electromagnetic sub-cascades are wider at low than at high primary energy in all investigated operation modes. For energies below around $200-300 \mathrm{GeV}$ the majority of the registered images contains less than 5 sub-cascades. The only false $\gamma$ and one $\pi^{0}$ candidates may be images with a small light contribution (less than $10 \%$ of the total SIZE) from the hadronic and muonic part of the shower (so called electromagnetic events) are presented as green histograms in Figure 1.

The primary energy distributions for trigger threshold 3 p.e. are presented in Figure 2. Solid histograms in Figure 2 present distributions obtained for both one $\pi^{0}$ and false $\gamma$. Dashed histograms were obtained for all triggered events. One may see that false $\gamma$ sub-cascade and one $\pi^{0}$ images correspond to the low proton primary energy in all observation modes. I have checked that at trigger threshold 3 p.e. approximately less than $5 \%$ of such events have a primary energy above $200 \mathrm{GeV}$ (in all investigated observation modes).

Supposing that proton shower, which have an impact parameter larger than simulated limit or the shower's inclination angle of the shower in respect to the telescope axis is larger than $5.5^{\circ}$, can not trigger telescope, one may estimate the expected number of trigger events, false $\gamma$ and one $\pi^{0}$ events for energies above $1 \mathrm{TeV}$ (upper simulated limit). The simple power law fit of the energy distribution tail was used as an extrapolation function. For all investigated trigger thresholds and observation modes, the fraction of false $\gamma$ (red lines in the Figure 4) and both false $\gamma$ and one $\pi^{0} \mathrm{im}$ ages (black lines in Figure 4) in the expected protonic background was calculated. Additionally the contribution of electromagnetic events (green lines in the Figure 4) has been calculated. Figure 4 shows how these fractions depend on the trigger threshold. The contribution of single sub-cascades events decreases with the trigger thresholds for all investigated observation modes. All possible events, which are triggered by one telescope only, contain from $35 \%$ to $14 \%$ both fasle $\gamma$ and one $\pi^{0}$ images at trigger thresholds of 2 p.e. and 5 p.e. respectively. The proton background, which triggered two telescopes, has lower contamination of investigated single sub-cascades images - approximately from $30 \%$ to $11 \%$. The same fraction calculated for last observation mode varies from $25 \%$ to $7 \%$.

One of the fundamental parameter which describe the image is SIZE (sum of all signals from pixels contained in the image). A few important image parameters (like WIDTH, LENGTH [14] etc.), which are used to the gamma/hadron separation depend on SIZE. SIZE is also a crucial parameter in the energy reconstruction procedure. As the shower may be detected simultaneously by more then one telescope, one may calculate an average SIZE per telescope. Figure 5 shows this average SIZE distributions of both false $\gamma$ and one $\pi^{0}$ images (solid histograms in the figure) and true $\gamma$ events (dashed histograms) obtained from MC simulations at the trigger thresholds 3 

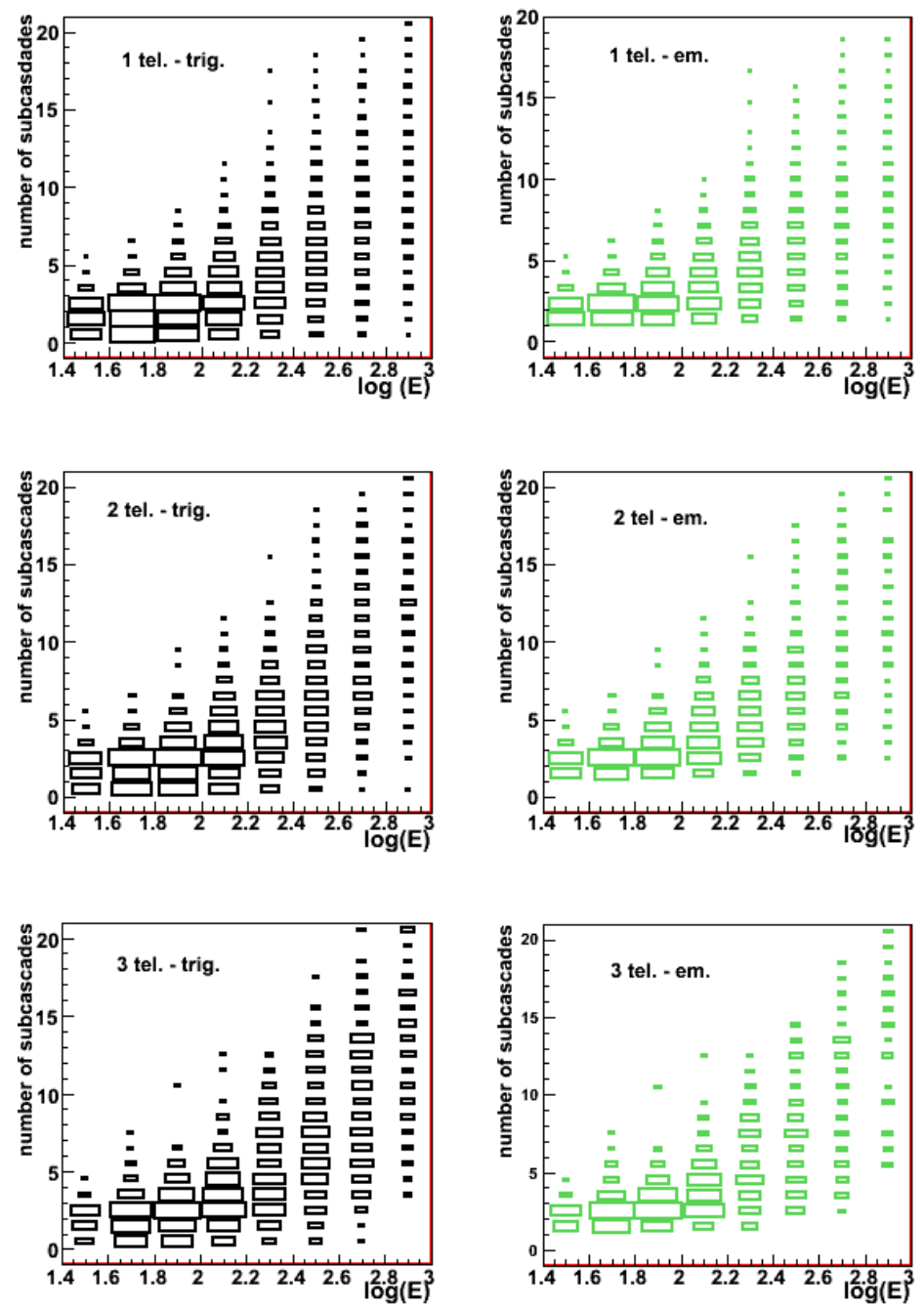

Figure 2: Number of electromagnetic sub-cascades which contribute to shower images versus the proton primary energy for the trigger threshold of 3 p.e. Green histograms (on the right side) show distributions obtained for images containing at least $90 \%$ of light from electrons and positrons.

p.e.. All distributions presented in Figure 5 are normalised to the expected number of showers within 60 seconds of the observation. The Crab spectrum [12] was used to calculate the number of real $\gamma$ events. The expected number of both false $\gamma$ and one $\pi^{0}$ images was estimated using the primary proton spectrum [15, 16]. The estimated number of both false $\gamma$ and one $\pi^{0}$ images strongly depends on the observation mode in all SIZE range - the lowest was obtained for III observation mode. The ratio of both false $\gamma$ and one $\pi^{0}$ images to that of primary $\gamma$-rays from the Crab Nebula direction depends on the SIZE bins. This ratio is decreasing with the average SIZE for all investigated observation modes. 


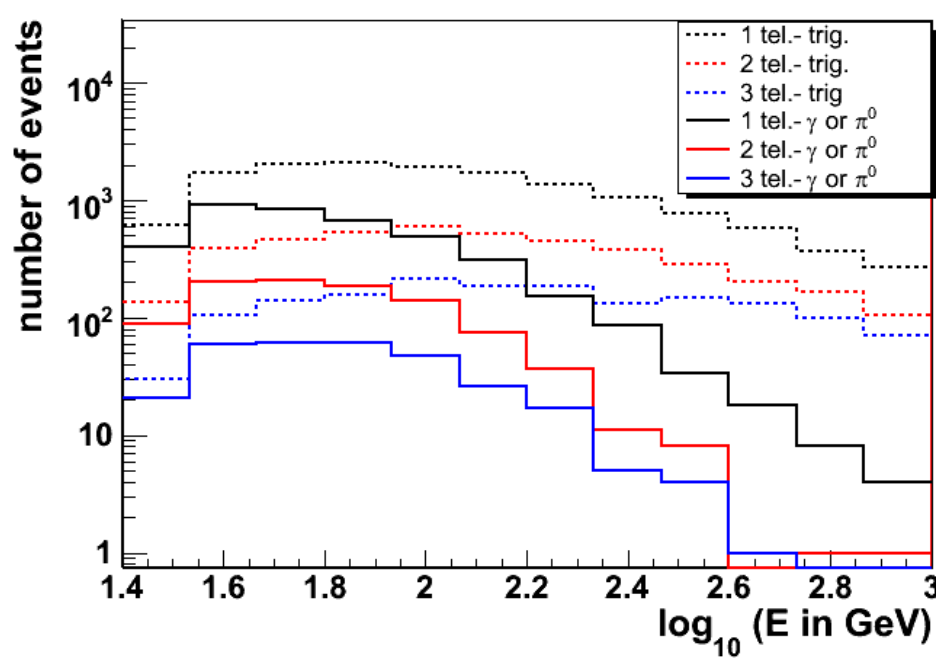

Figure 3: Energy distribution for proton events (trigger threshold of 3 p.e.). Solid histograms show distributions obtained from false $\gamma$ and one $\pi^{0}$ events.

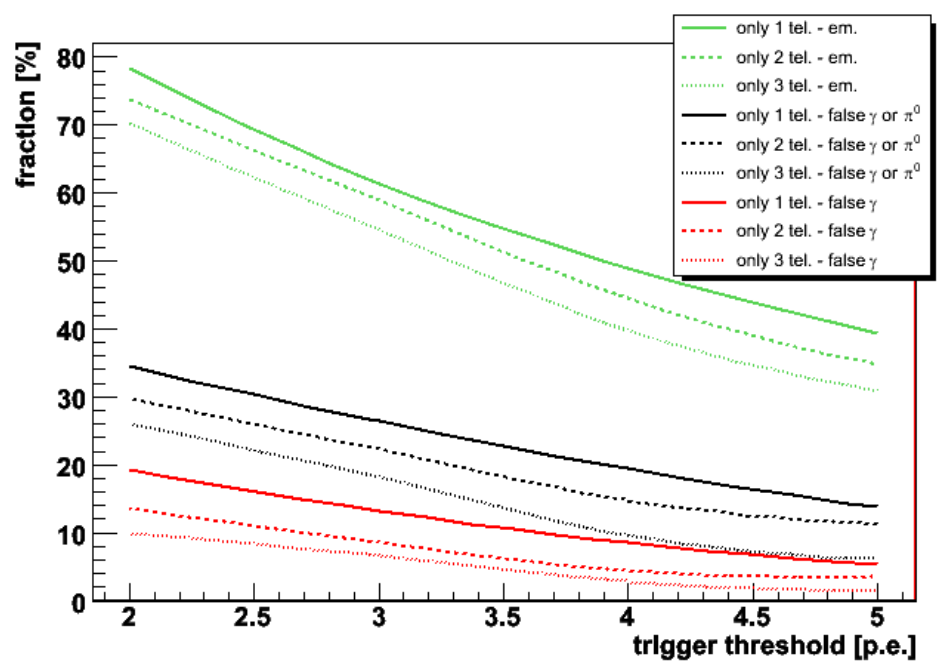

Figure 4: Fraction of the interesting events in the total protonic background as a function of the trigger threshold

\section{Conclusions}

The distribution of the number of electromagnetic sub-cascades (which contribute in the shower image) strongly depends on the proton primary energy. The occurrence of investigated background (false $\gamma$ and one $\pi^{0}$ ) is mostly caused by low energy proton induced showers. Approximately less than $5 \%$ of such events have a primary energy above $200 \mathrm{GeV}$ (at trigger threshold 3 of p.e.) in all investigated trigger modes. The fraction of hardly reducible background is decreasing with the number of triggered telescopes (from 1 to 3 ) at fixed trigger threshold. The proton background (which triggered three telescopes only) contains $25 \%$ of false gamma and one $\pi^{0}$ events at a trigger 


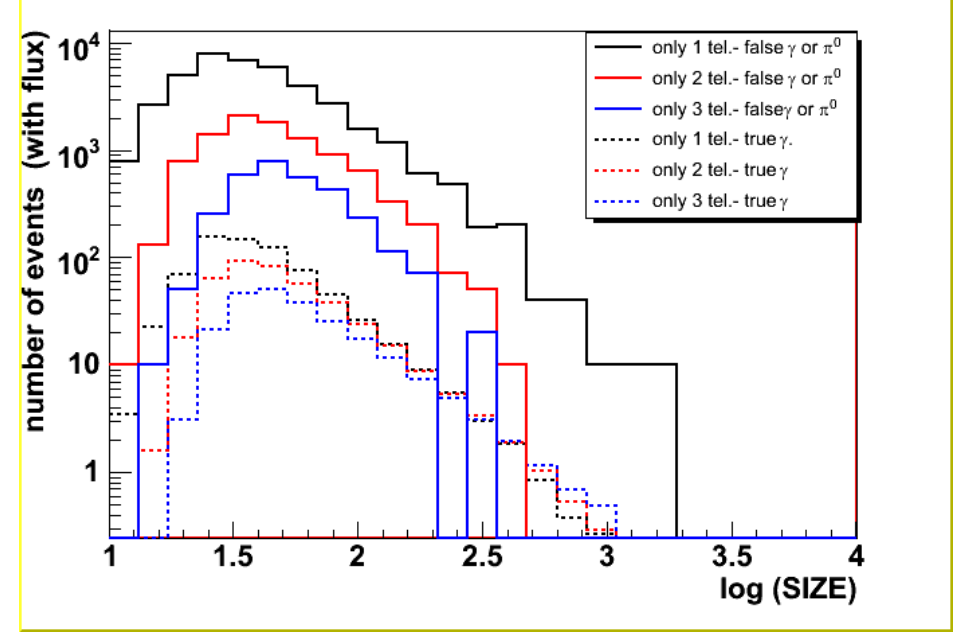

Figure 5: Average SIZE (per telescope) distributions for proton showers; trigger threshold 3 p.e. Histograms are normalised to the number of events expected within 60 second of observation.

threshold of 2 p.e. which decreases to $7 \%$ at a trigger threshold of 5 p.e. In all investigated observation modes, the fraction a single sub-cascades (both of false gamma and one $\pi^{0}$ ) images in the proton background strongly depends on the average SIZE range. This fraction is much higher for lower SIZES than for higher SIZES.

\section{References}

[1] Sobczynska D. 2007, J.Phys.G: Nucl. Part. Phys. 34, 2279

[2] Sobczynska D. 2009, J.Phys.G: Nucl. Part. Phys. 36, 125201

[3] Sobczynska D. 2009, Proc. 31th Int. Cosmic Ray Conf.(Lodz), ID=1112

[4] Heck D. et al 1998, Technical Report FZKA 6019 (Forschungszentrum Karlsruhe)

[5] Knapp J. and Heck D. 2004, EAS Simulation with CORSIKA: A User's Manual

[6] Barrio J. A. et al 1998, The MAGIC Telescope Design Study (Munich)

[7] Baixeras C. et al 2004, Nucl. Instrum. Methods Phys. Res. A 518, 188

[8] Albert J. et al 2005, Astropart Phys 23, 493

[9] Carmona E. et al 2007, Proc. 30th Int. Cosmic Ray Conf. (Merida, Mexico) vol 3, 1373

[10] Mirzoyan R. and Lorenz E. 1994, MPI-PhE/94-35 preprint (Munich)

[11] Sokolsky P. 1989, "Introduction to Ultrahigh Energy Cosmic Ray Physics" Addison-Wesley

[12] Wagner R. et al 2005, Proc. 29th Int. Cosmic Ray Conf. (Pune) vol 4, 163

[13] Aharonian F. et al 2004, Astrophys. J. 314897

[14] Hillas A. M. 1985, Proc. 19th Int. Cosmic Ray Conf. (La Jolla) vol 3, 445

[15] Haino S. et al 2004 Phys. Lett. B 59435

[16] Hareyama M. and Shibata T. 2005, Int. J. Modern Phys. A 29, 6769 